\title{
Short communication: Genome-wide identification of new reference genes for reverse-transcription quantitative PCR in Streptococcus thermophilus based on RNA-sequencing analysis
}

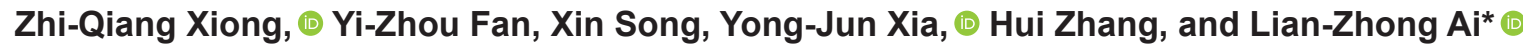 \\ Shanghai Engineering Research Center of Food Microbiology, School of Medical Instrument and Food Engineering, \\ University of Shanghai for Science and Technology, Shanghai 200093, China
}

\begin{abstract}
Streptococcus thermophilus, one of the most important industrial lactic acid bacteria, is widely used for the production of fermented dairy products such as yogurt and cheese. The accuracy of gene expression-based analyses (e.g., reverse-transcription quantitative real-time PCR) relies heavily on the selection of reliable reference genes (RG), which provides the basis for correctly interpreting expression data. However, many traditional RG are not stably expressed in different systems. Here we used RNA-sequencing to systematically investigate gene expression variation at the genome scale and identify more stable RG in $S$. thermophilus. In total, 21 putative candidate RG were identified with variation coefficient values $<10.0$ based on the expression of all 1,911 genes under 4 different experimental conditions. We selected and validated $12 \mathrm{RG}$ chosen from transcriptomes by using reverse-transcription quantitative real-time PCR, and ranked their expression stability by statistical algorithms geNorm and NormFinder. Compared with traditional RG $16 S$ rRNA, genes encoding glycinetRNA ligase subunit $\beta$ GlyS and fatty acid-binding protein DegV were more stable under all 4 treatments, which have never been used as RG in S. thermophilus. Our finding provides the foundation for more precise analysis of gene expression in S. thermophilus and other lactic acid bacteria species.
\end{abstract}

Key words: Streptococcus thermophilus, RNA sequencing, reverse-transcription quantitative real-time PCR, reference genes

\section{Short Communication}

Streptococcus thermophilus is one of the generally recognized as safe lactic acid bacteria (LAB) with

Received April 7, 2020.

Accepted June 22, 2020.

*Corresponding author: ailianzhong1@126.com nonpathogenic, fermentative, and facultative anaerobic characteristics (Xiong et al., 2019a). As an important homo-fermentative streptococcal species of LAB, $S$. thermophilus is widely used for dairy industry such as yogurt and cheese production (Sørensen et al., 2016). The traits of $S$. thermophilus relevant to dairy products include lactose utilization, rapid acidification, improvement of the texture and flavor properties, as well as bacteriocin and exopolysaccharide synthesis (Xiong et al., 2019b). Streptococcus thermophilus prefers lactose over glucose as a carbon and energy source in dairy fermentation (Anbukkarasi et al., 2014), which can assist people with lactose intolerance to fully absorb milk nutrients. In addition, S. thermophilus has various probiotic functions (e.g., modulation of intestinal microbiota; Cui et al., 2016).

Reverse-transcription quantitative real-time PCR (RT-qPCR) is currently the most frequently used technique to quantify gene expression in diverse biological systems (Pombo et al., 2017). Selection of reliable reference genes (RG) is critical to affect the accuracy and interpretation of RT-qPCR results. Normalization of target gene expression by using invalidate RG will result in the questionable or even misleading results. Ideal RG are internal controls that should show minimal or no variation of their expression levels in various experimental conditions (Wu et al., 2017). Traditionally, RG are the most commonly used housekeeping genes that are presumed to have relatively stable expression in biological systems under different environments (Xia et al., 2014). However, recent studies have suggested that the transcript levels of these traditional RG are not as stable as we thought under different experimental conditions (Xia et al., 2014; Pombo et al., 2017; Wang et al., 2017). To data, most studies of gene expression by RT-qPCR in bacteria have still arbitrarily selected certain conventional RG without an evaluation of stability or even without an explanation (Krzyżanowska et al., 2019). Also, no in-depth analysis is available of stable RG in S. thermophilus, which limits accurately quantitative analysis of gene expression. 
With the rapid development of high-throughput sequencing, RNA-sequencing (RNA-seq) has recently been employed to uncover more reliable RG for RTqPCR studies in various species under different experimental conditions (Xia et al., 2014; Pombo et al., 2017; Wang et al., 2017; Nong et al., 2019). Hence, to identify new RG with lower variation for RT-qPCR, we used RNA-seq to systematically assess gene expression variation of $S$. thermophilus under different treatments. Wild-type $S$. thermophilus S-3 (galactose-positive) and AR333 (galactose-negative) in this study were isolated from traditional Chinese dairy products (Xiong et al., 2019b); they were cultivated in both GM17 and LM17 liquid medium at $37^{\circ} \mathrm{C}$ without shaking for 24 h. Accordingly, the 4 experimental conditions were (1) strain S-3 grown in GM17 medium, (2) strain S-3 grown in LM17 medium, (3) strain AR333 grown in GM17 medium, and (4) strain AR333 grown in LM17 medium. According to our previous report (Kong et al., 2019), total RNA of $S$. thermophilus samples was extracted using Trizol reagent (Thermo Fisher Scientific, Waltham, MA) and ribosomal RNA was removed by a Ribo-Zero Magnetic kit (Epicenter, Madison, WI). The cDNA libraries were prepared using the TruSeq RNA Library Preparation kit (Illumina, San Diego, CA) and sequenced on the Illumina Hiseq 4000 platform by Shanghai Majorbio Bio-pharm Technology Co. Ltd. (Shanghai, China). The strength of gene expression could be reflected by fragments per kilobase of gene model per million mapped reads (FPKM), which was calculated using the FPKM function from edgeR v.3.4.0.3 (Kong et al., 2019). Based on the reference genome data of $S$. thermophilus LMD-9 (NC_008532), the transcriptional profiling of all genes in strains S-3 and AR333 was sorted by FPKM under the 2 target culturing conditions (GM17 and LM17 media). To rank the transcript level stability of the predicted 1,911 genes, we calculated the average reads per kilobase of transcript per million mapped reads (RPKM) value, standard deviations, and variation coefficient (VC, the ratio between SD and the average RPKM of each gene expression) using all the RPKM obtained from the above 4 treatments. We screened 21 genes that satisfied with $\mathrm{VC}<0.1$ and ordered the gene list according to the lowest VC value (Table 1). For this set of genes, VC ranged from $4.10 \%$ to $9.88 \%$. The lower VC indicated more stable gene expression across all the conditions. Interestingly, none of 21 selected genes had been used previously as RG in S. thermophilus. Primarily housekeeping genes such as $16 S$ rRNA (16S ribosomal RNA), GAPDH (glyceraldehyde-3-phosphate dehydrogenase), and recA (DNA recombination/repair protein) have been widely used as traditional RG in bacteria. However, higher VC values of $16 S$ rRNA (19.9\%), GAPDH
(30.8\%), and recA (43.4\%) across all the treatments suggested the possibility of low gene expression stability of traditional RG (Table 1). The $16 S$ rRNA gene had a low $\mathrm{VC}$ value in comparison with the other $2 \mathrm{RG}$. Moreover, the $16 S r R N A$ gene is the most commonly used RG in S. thermophilus (Anbukkarasi et al., 2014). Hence, we selected $16 S r R N A$ as the control for further validation by $\mathrm{RT}-\mathrm{qPCR}$.

To assess the possibility of 21 candidate genes as $\mathrm{RG}$, we extracted the nucleotide sequence of each gene from the genome data of LMD-9 (Supplemental File S1; https://doi.org/10.3168/jds.2020-18672) and designed primers (Supplemental Table S1; https://doi.org/10 .3168/jds.2020-18672) using Vector NTI Advance software (ver. 11.5, Invitrogen, Carlsbad, CA). The method of RNA extraction and RT-qPCR was previously described by Xiong et al. (2020). The specificity of the amplification for all the pair of primers was evaluated by melting curves and agarose gel electrophoresis. If there was not a single peak in the melting curve or no single amplification product in the $2 \%$ agarose gel, we discarded that pair of primers (Supplemental Table S1). We used cDNA dilutions (1, 1:10, 1:100, 1:1,000, and 1:10,000) for RT-qPCR. Amplification efficiency $(\boldsymbol{E})$ was measured as $10^{-1 / \text { slope }}$ and expressed in percentage (Supplemental Table S1). Except for abnormally high $E$ values $(>150 \%)$ of the primers of $S T E R \_R S 01160, \quad S T E R \_R S 06475, \quad S T E R \_R S 01675$, $S T E R \_R S 06355$, and $S T E R \_R S 08540$, other primers showed good amplification with $E$ values ranging from $80.7 \%$ to $129.9 \%$. We also evaluated the performance of these candidate genes under the above 4 treatments by cycle amplification values (Cq, Figure 1). Average $\mathrm{Cq}$ values ranging from 18.7 (STER_RS10000) to 31.7 (STER_RS05195) suggested that most of the genes (except for STER_RS05195) have suitable Cq values within the recommended values (15-30) for RTqPCR RG (Pombo et al., 2017). According to $E$ and Cq values, we abandoned the genes STER_RS01160, STER_RS06475, STER_RS01675, STER_RS06355, $S T E R \_R S 08540$, and STER_RS05195 as candidate $\mathrm{RG}$.

To evaluate the expression stability of our selected genes, we used 2 different tools to analyze the RT-qPCR data across all 4 conditions. The geNorm software (Vandesompele et al., 2002) first determined the average expression stability value $M$ (the average pairwise variation of a particular gene with all other control genes) under various experimental conditions. A gene with a lower $M$ value is more stable. All the analyzed genes showed lower $M$ values than that of the common RG $16 S$ rRNA ( $M=3.83$, Figure $2 \mathrm{~A}$ and Supplemental Table S2; https://doi.org/10.3168/jds.2020 -18672). The most stable genes were $S T E R \_R S 10085$, 


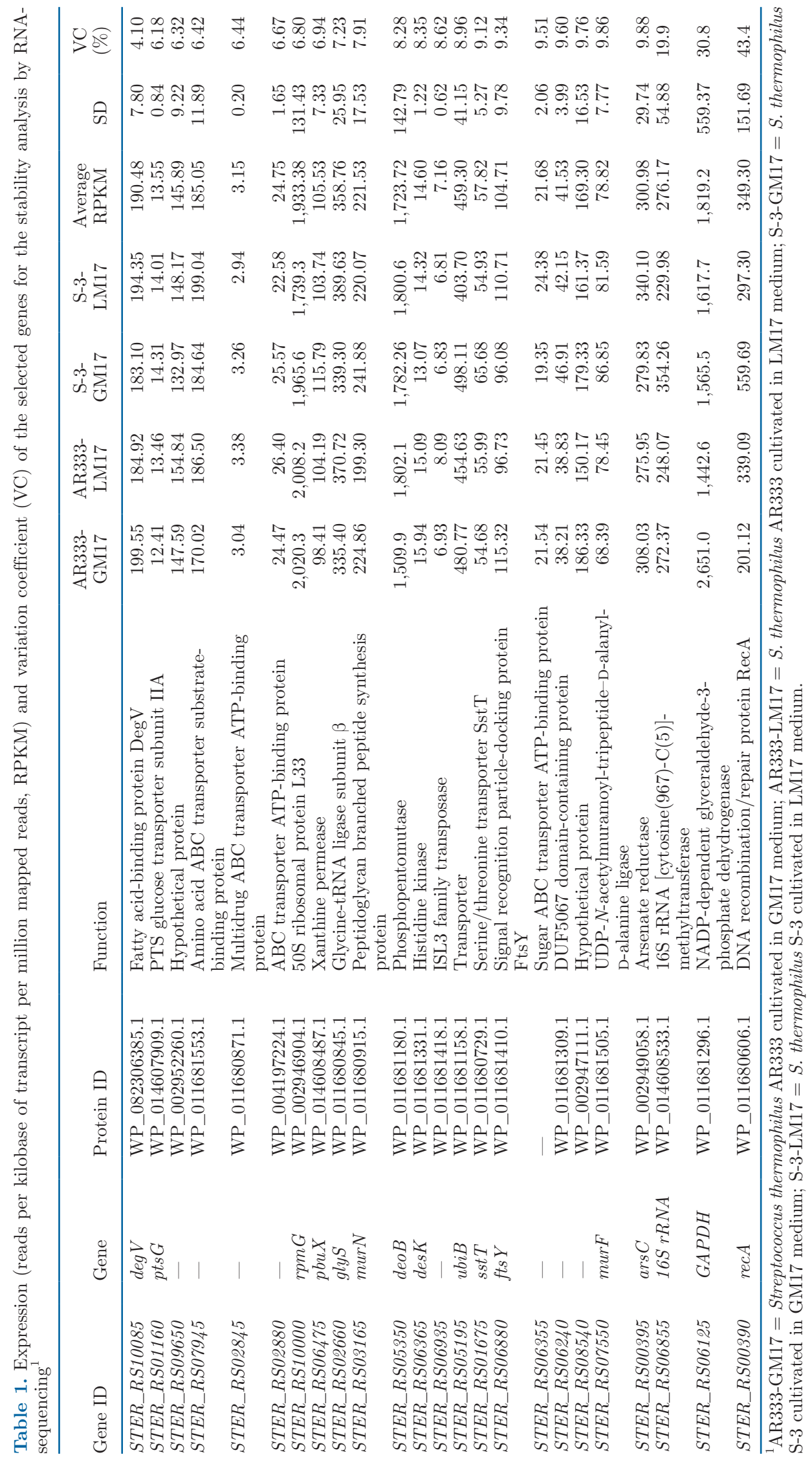




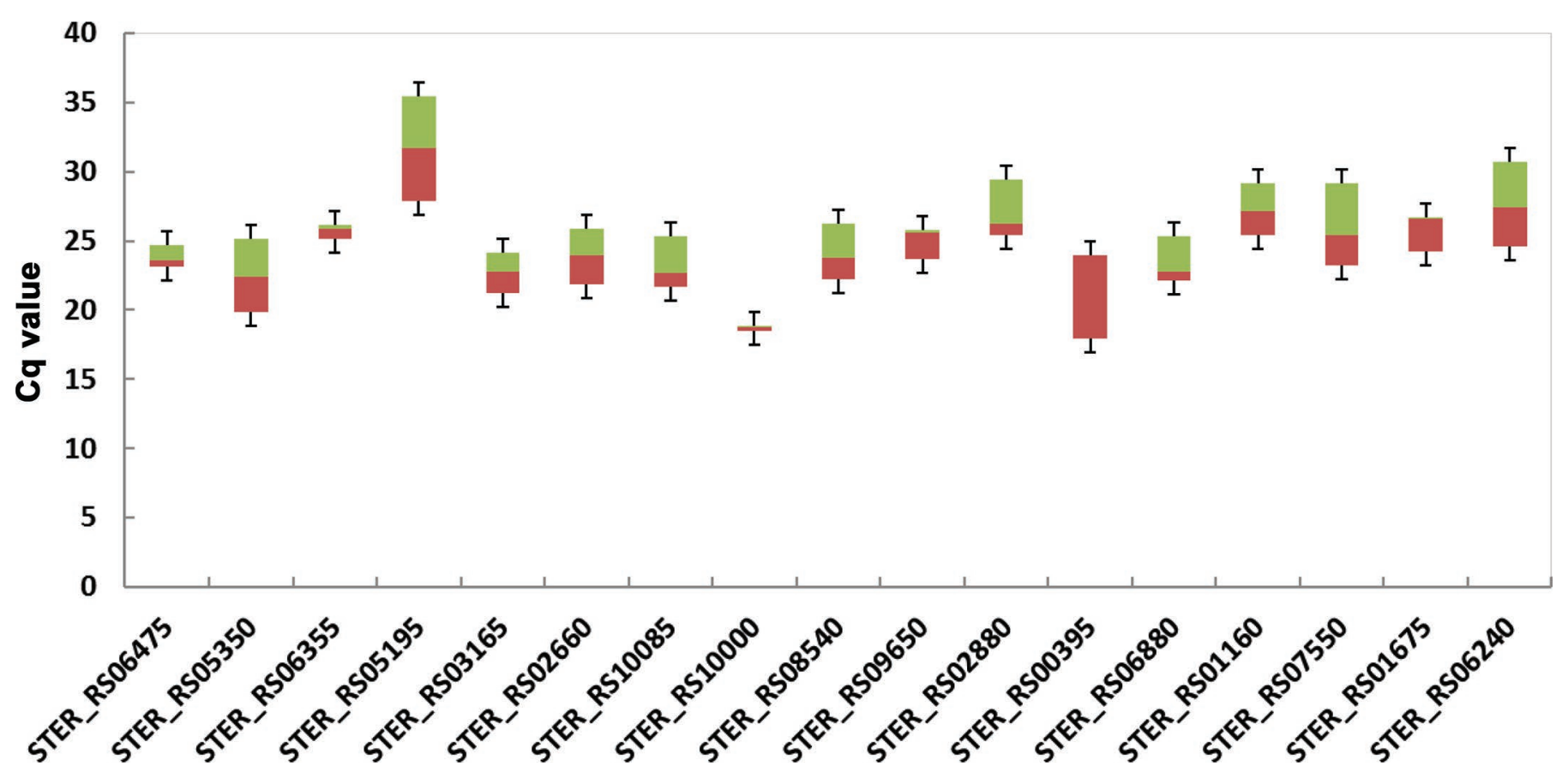

Figure 1. Cycle quantification $(\mathrm{Cq})$ values of 21 candidate genes and $16 S r R N A$. Box and whisker plot graph showing Cq values of each selected gene in all the experimental conditions. Upper and lower edges of boxes indicate the 25th and 75th percentiles. The lines across the boxes depict the median. Whisker caps represent the minimum and maximum values.

STER_RS02660, and STER_RS03165 with $M$ values of $1.43,1.44$, and 1.48 , respectively, by the geNorm algorithm. We further investigated expression stability of these selected genes with NormFinder software (Andersen et al., 2004), which can also calculate $M$ index with taking into account the intragroup and intergroup variations. Like geNorm, NormFinder analysis presented the similar result that all candidate genes have lower $M$ values than $16 S$ rRNA $(M=2.55$, Figure 2B and Supplemental Table S2). The most reliable genes were also $S T E R \_R S 02660, S T E R \_R S 10085$, and $S T E R \_R S 03165$ with $M$ values of $0.13,0.18$, and 0.28 , respectively. Although the results of gene expression stability obtained from geNorm and NormFinder were largely consistent, a few discrepancies of ranking orders were observed by these 2 statistical programs based on different algorithms. Pombo et al. (2017) recommended considering the complementarity between these methods and globally analyzing the results. Thus, the arithmetical mean of the ranking value by different algorithms was calculated for each candidate gene. As expected, STER_RS02660 (glyS) and STER_RS10085 $(\operatorname{deg} V)$ were sorted as the most stable genes with a mean value of 1.5 (Supplemental Table S2). Thus, we recommend $g \operatorname{ly} S$ and $\operatorname{deg} V$ as the better choices for universal RG in $S$. thermophilus.

The gold standard of gene expression quantification is RT-qPCR (DeLorenzo and Moon, 2018), which highly depends on RG for the accuracy and reliability of the data. Unfortunately, no in-depth analysis of stable RG has been performed in $S$. thermophilus and other LAB species. In this work, we used RNA-seq as a new approach for genome-wide identification of new stable RG in $S$. thermophilus. To our knowledge, this is the first time of identifying RG in $S$. thermophilus. A set of new candidate RG with low $\mathrm{VC}$ values was selected from the expression of all 1,911 genes in the genome and further validated by RT-qPCR. Based on the analysis of expression stability by geNorm and NormFinder algorithms, all candidate genes were more stable than traditional RG $16 S \mathrm{rRNA}$ under 4 experimental conditions. Our results strongly supported the use of highthroughput transcriptomic data for mining the highly stable RG in RT-qPCR experiments (Xia et al., 2014; Pombo et al., 2017; Wang et al., 2017), which provides a valuable starting point for more precise results of gene expression in $S$. thermophilus and other LAB.

\section{ACKNOWLEDGMENTS}

This work was supported by the National Natural Science Foundation of China (Beijing; grant no. 31871776, 31972101, and 31771956), Natural Science Foundation of Shanghai (grant no. 18ZR1426800, China), Shanghai Agriculture Applied Technology Development Program (grant no. 2019-02-08-00-07-F01152, China), and 


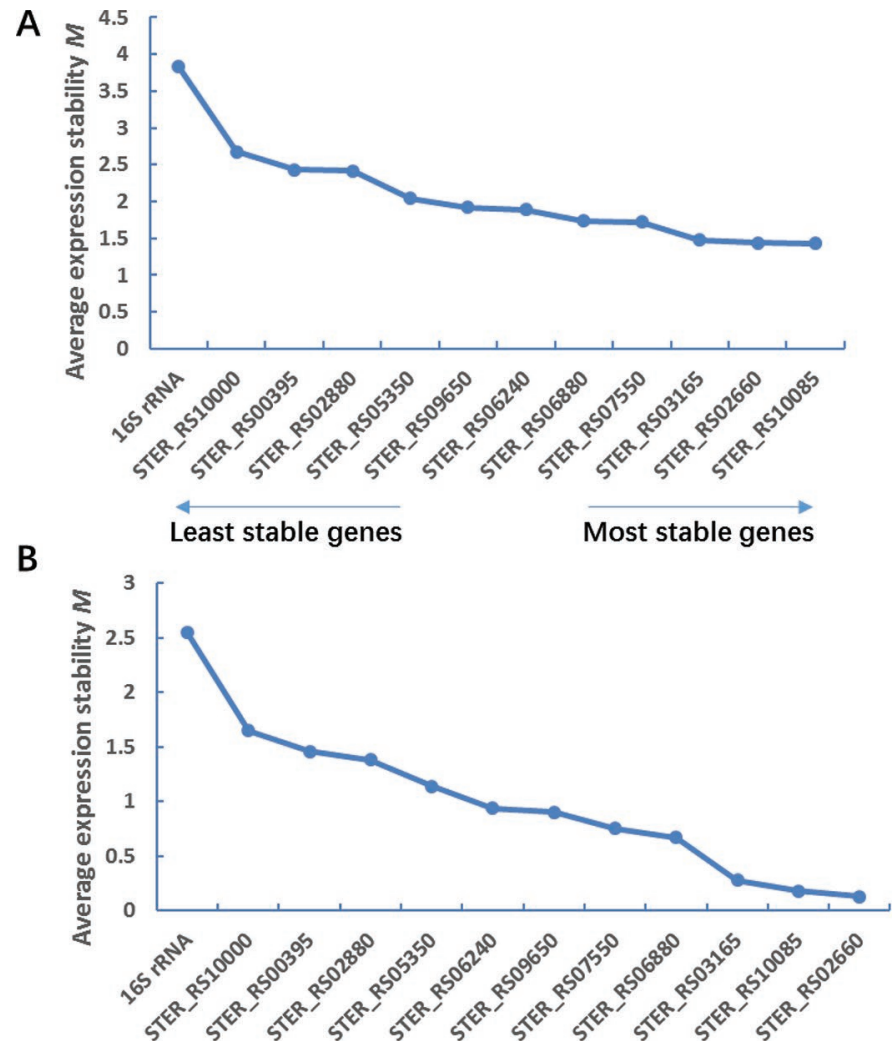

Figure 2. Expression stability of selected genes in Streptococcus thermophilus using geNorm (A; Vandesompele et al., 2002) and NormFinder (B; Andersen et al., 2004) analysis. The analysis was performed using expression data from all biological replicates and treatments. The $M$ value represents the average pairwise variation of a particular gene in comparison with all other control genes.

Shanghai Engineering Research Center of Food Microbiology (grant no. 19DZ2281100, China). The authors have not stated any conflicts of interest.

\section{REFERENCES}

Anbukkarasi, K., D. K. Nanda, T. UmaMaheswari, T. Hemalatha, P. Singh, and R. Singh. 2014. Assessment of expression of Leloir pathway genes in wild-type galactose-fermenting Streptococcus thermophilus by real-time PCR. Eur. Food Res. Technol. 239:895903. https://doi.org/10.1007/s00217-014-2286-9.

Andersen, C. L., J. L. Jensen, and T. F. Orntoft. 2004. Normalization of real-time quantitative reverse transcription-PCR data: A model-based variance estimation approach to identify genes suited for normalization, applied to bladder and colon cancer data sets. Cancer Res. 64:5245-5250. https://doi.org/10.1158/0008-5472 .CAN-04-0496.

Cui, Y., T. Xu, X. Qu, T. Hu, X. Jiang, and C. Zhao. 2016. New insights into various production characteristics of Streptococcus thermophilus strains. Int. J. Mol. Sci. 17:1701. https://doi.org/10 $.3390 /$ ijms17101701.
DeLorenzo, D. M., and T. S. Moon. 2018. Selection of stable reference genes for RT-qPCR in Rhodococcus opacus PD630. Sci. Rep. 8:6019. https://doi.org/10.1038/s41598-018-24486-w.

Kong, L. H., Z. Q. Xiong, X. Song, Y. J. Xia, N. Zhang, and L. Z. Ai. 2019. Characterization of a panel of strong constitutive promoters from Streptococcus thermophilus for fine-tuning gene expression. ACS Synth. Biol. 8:1469-1472. https://doi.org/10.1021/acssynbio $.9 \mathrm{~b} 00045$.

Krzyżanowska, D. M., A. Supernat, T. Maciag, M. Matuszewska, and S. Jafra. 2019. Selection of reference genes for measuring the expression of aiiO in Ochrobactrum quorumnocens A44 using RTqPCR. Sci. Rep. 9:13129. https://doi.org/10.1038/s41598-019 $-49474-6$

Nong, Q., Y. Yang, M. Zhang, M. Zhang, J. Chen, S. Jian, H. Lu, and K. Xia. 2019. RNA-seq-based selection of reference genes for RTqPCR analysis of pitaya. FEBS Open Bio 9:1403-1412. https://doi .org/10.1002/2211-5463.12678.

Pombo, M. A., Y. Zheng, Z. Fei, G. B. Martin, and H. G. Rosli. 2017. Use of RNA-seq data to identify and validate RT-qPCR reference genes for studying the tomato-Pseudomonas pathosystem. Sci. Rep. 7:44905. https://doi.org/10.1038/srep44905.

Sorensen, K. I., M. Curic-Bawden, M. P. Junge, T. Janzen, and E. Johansen. 2016. Enhancing the sweetness of yoghurt through metabolic remodeling of carbohydrate metabolism in Streptococcus thermophilus and Lactobacillus delbrueckii ssp. bulgaricus. Appl. Environ. Microbiol. 82:3683-3692. https://doi.org/10.1128/AEM .00462-16.

Vandesompele, J., K. De Preter, F. Pattyn, B. Poppe, N. Van Roy, A. De Paepe, and F. Speleman. 2002. Accurate normalization of real-time quantitative RT-PCR data by geometric averaging of multiple internal control genes. Genome Biol 3:RESEARCH0034.

Wang, H., X. Zhang, Q. Liu, X. Liu, and S. Ding. 2017. Selection and evaluation of new reference genes for RT-qPCR analysis in Epinephelus akaara based on transcriptome data. PLoS One 12:e0171646. https://doi.org/10.1371/journal.pone.0171646.

Wu, B. Y., J. R. Ye, L. Huang, L. M. He, and D. W. Li. 2017. Validation of reference genes for RT-qPCR analysis in Burkholderia pyrrocinia JK-SH007. J. Microbiol. Methods 132:95-98. https:// doi.org/10.1016/j.mimet.2016.10.004.

Xia, W., A. S. Mason, Y. Xiao, Z. Liu, Y. Yang, X. Lei, X. Wu, Z. Ma, and M. Peng. 2014. Analysis of multiple transcriptomes of the African oil palm (Elaeis guineensis) to identify reference genes for RT-qPCR. J. Biotechnol. 184:63-73. https://doi.org/10.1016/ j.jbiotec.2014.05.008

Xiong, Z. Q., L. H. Kong, P. F. Lai, Y. J. Xia, J. C. Liu, Q. Y. Li, and L. Z. Ai. 2019a. Genomic and phenotypic analyses of exopolysaccharide biosynthesis in Streptococcus thermophilus S-3. J. Dairy Sci. 102:4925-4934. https://doi.org/10.3168/jds.2018-15572.

Xiong, Z. Q., L. H. Kong, H. L. Meng, J. M. Cui, Y. J. Xia, S. J Wang, and L. Z. Ai. 2019b. Comparison of gal-lac operons in wildtype galactose-positive and -negative Streptococcus thermophilus by genomics and transcription analysis. J. Ind. Microbiol. Biotechnol. 46:751-758. https://doi.org/10.1007/s10295-019-02145-x.

Xiong, Z. Q., Y. Y. Wei, L. H. Kong, X. Song, H. X. Yi, and L. Z. Ai. 2020. Short communication: An inducible CRISPR/dCas9 gene repression system in Lactococcus lactis. J. Dairy Sci. 103:161-165. https://doi.org/10.3168/jds.2019-17346.

\section{ORCIDS}

Zhi-Qiang Xiong (ํ) https://orcid.org/0000-0002-7984-3662

Yong-Jun Xia ๑ https://orcid.org/0000-0002-2727-2730

Lian-Zhong Ai ๑ https://orcid.org/0000-0002-6681-9102 